\title{
Outcomes after primary and repeat thermal ablation of hepatocellular carcinoma with or without liver transplantation
}

\author{
Christiaan M. C. Serbanescu-Kele Apor de Zalán ${ }^{1}$ · Simeon J. S. Ruiter ${ }^{1} \cdot$ Aad P. van den Berg $^{2} \cdot$ Jan Pieter Pennings ${ }^{3}$. \\ Koert P. de Jong ${ }^{1}$
}

Received: 10 August 2021 / Revised: 26 November 2021 / Accepted: 10 December 2021 / Published online: 8 February 2022

(c) The Author(s) 2022

\begin{abstract}
Objectives Thermal ablation (TA) is an established treatment for early HCC. There is a lack of data on the efficacy of repeated TA for recurrent HCC, resulting in uncertainty whether good oncologic outcomes can be obtained without performing orthotopic liver transplantation (OLTx). This study analyses outcomes after TA, with a special focus on repeat TA for recurrent HCC, either as a stand-alone therapy, or in relationship with OLTx.

Methods Data from a prospectively registered database on interventions for HCC in a tertiary hepatobiliary centre was completed with follow-up until December 2020. Outcomes studied were rate of recurrence after primary TA and after its repeat interventions, the occurrence of untreatable recurrence, OS and DSS after primary and repeat TA, and complications after TA. In cohorts matched for confounders, OSS and DSS were compared after TA with and without the intention to perform OLTx. Results After TA, 100 patients (56.8\%) developed recurrent HCC, of whom $76(76.0 \%)$ underwent up to four repeat interventions. During follow-up, $76.7 \%$ of patients never developed a recurrence unamenable to repeat TA or OLTx. OS was comparable after primary TA and repeat TA. In matched cohorts, OS and DSS were comparable after TA with and without the intention to perform OLTx.

Conclusions We found TA to be an effective and repeatable therapy for primary and recurrent HCC. Most recurrences can be treated with curative intent. There are patients who do well with TA alone without ever undergoing OLTx.

Key Points

- Recurrent HCC after primary TA can often be treated effectively with repeat TA. Survival after repeat TA is comparable to primary $T A$.

- In matched cohorts, outcomes after TA with and without subsequent waitlisting for OLTx are comparable.

- There are patients who do well for many years with primary and repeat TA alone; some despite multiple recurrences.
\end{abstract}

Keywords Carcinoma, hepatocellular $\cdot$ Liver Neoplasms $\cdot$ Hyperthermia, induced $\cdot$ Liver Transplantation $\cdot$ Neoplasm recurrence, local

Christiaan M. C. Serbanescu-Kele Apor de Zalán

c.m.c.serbanescu-kele@umcg.nl; cornel_d_S@hotmail.com

1 Department of Hepato-Pancreatico-Biliary Surgery and Liver Transplantation, University Medical Centre Groningen, University of Groningen, Hanzeplein 1, 9713 GZ Groningen, The Netherlands

2 Department of Gastroenterology, University Medical Centre Groningen, University of Groningen, Hanzeplein 1, 9713 GZ Groningen, The Netherlands

3 Department of Radiology, University Medical Centre Groningen, University of Groningen, Hanzeplein 1, 9713 GZ Groningen, The Netherlands

\section{Abbreviations}

AFP Alpha-foetoprotein

CT Computed tomography

DSS Disease-specific survival

HCC Hepatocellular carcinoma

HR Hepatic resection

OLTx Orthotopic liver transplantation

OS Overall survival

RFS Recurrence-free survival

TA Thermal ablation 


\section{Introduction}

Hepatocellular carcinoma (HCC) is a major cause of global morbidity and mortality [1], with an rising incidence in the Western world due to an increasing prevalence of steatohepatitis [2]. Staged according to the BCLC classification, only very early (single tumour, $<2 \mathrm{~cm}$ ) and early $\mathrm{HCC}$ (up to three tumours, $<3 \mathrm{~cm}$ each) are generally considered curable [3]. Curative treatment options include thermal ablation (TA, which includes microwave ablation and radiofrequency ablation), hepatic resection (HR), and orthotopic liver transplantation (OLTx) [4]. The latter is generally considered to yield the best recurrence-free survival [5], but is limited by a scarcity in available donor organs, and considerable procedural morbidity and mortality [6]. Conversely, liver-sparing treatment modalities are plagued by higher rates of tumour recurrence [3]. Typically, initial therapy is with either HR or TA, after which some patients may eventually undergo OLTx [7].

Controversy still shrouds the optimal treatment strategy for early and very early HCC [8]. It has been shown that carefully performed TA can result in excellent locoregional control of primary HCC [9]. Data on outcomes after repeat TA for recurrence following succesful primary TA, however, is sparse. Even more controversial is the timing of OLTx after successful TA in patients with preserved liver function [8]. Should TA be considered 'only' as a stop-gap measure until curative treatment is performed in the form of OLTx, or can TA itself, in selected cases, be considered a definitive therapy, provided that strict follow-up imaging is performed, in order to detect possible recurrences at an early stage? We aim to contribute to this debate by presenting our data on outcomes after primary and repeat TA, with and without subsequent OLTx, in a large tertiary referral centre.

\section{Patients and methods}

The present study focuses on outcomes after primary and repeat TA for HCC, with and without subsequent OLTx. In our centre, all cases of HCC are discussed in a multidisciplinary team. From 2000 onward, percutaneous TA was performed in a joint effort by specialised interventional radiologists and hepatobiliary surgeons. All patients who underwent TA, HR, or OLTx for HCC were prospectively entered into a database. In this study, we analysed patients who underwent primary TA between January 2000 and December 2019. Follow-up was completed up to December 2020. Outcomes studied were recurrence rate after primary TA and after its repeat interventions, the occurrence of untreatable recurrence, OS and DSS after primary TA and repeat interventions, and complications after TA (graded according to Clavien-Dindo [10]). We reconstructed patient-specific treatment paths, registering treatment modalities, time of recurrence (if any), and follow-up status. In order to compare survival without bias from tumour stage, age or comorbidity, we analysed patients who had been waitlisted for OLTx at any time after primary TA ("intention-to-performOLTX"), and compared them to a cohort of patients matched for age ( $<70$ years), tumour size and number (within Milan criteria) and comorbidity (ASA $<3$; no severe cardiopulmonary comorbidity), who upon revision of charts by a liver transplant surgeon would have been eligible for OLTx from a medical point of view, but who were never waitlisted due to other reasons ("eligible, non-waitlisted").

All instances of percutaneous TA were performed under computer tomography $(\mathrm{CT})$ guidance while patients were under general anesthesia, and mechanically ventilated. Image acquisition and needle positioning were performed under controlled apnea during an expiratory breath hold, allowing for constant anatomical relations. We believe this to be critical to our TA workflow, as accurate needle positioning is a prerequisite of curative locoregional treatment [11]. All patients underwent a contrast-enhanced CT scan one week after TA, to assess completeness of ablation and to obtain a baseline scan for optimal comparison during follow-up. If this scan revealed an inadequate ablation zone, patients underwent completion ablation as soon as possible. This was defined as incomplete ablation, and the completion ablation was not counted as a separate procedure, in accordance with the standardised reporting criteria proposed by Ahmed et al. [12]. Follow-up consisted of 4-monthly contrast-enhanced CT scans, laboratory workup, including serum alpha-foetoprotein (AFP) and, where indicated, MRI or other imaging studies. We defined recurrence as any lesion arising after successful treatment (as evidenced by satisfactory margins on the CT scan at day 7), either with the radiological characteristics of HCC, or based on histopathological examination. Time of recurrence was defined as the timepoint at which any such lesion was first detected. Where missing, dates and causes of death were registered by contacting patients' general practitioners. In case of doubt, death was assumed to be due to HCC. Survival was calculated separately from the date of primary intervention, and from the date of any subsequent interventions. This eliminates the time span between interventions, which might erroneously be interpreted as an increase in survival when multiple interventions have been performed (comparable to "lead-time" bias). Recurrence-free survival (RFS) was calculated from the time of intervention to recurrence of HCC; DSS was calculated from the time of intervention to the time of death from HCC.

Statistical analysis was performed with IBM SPSS (version 23). A $\chi^{2}$ test was used for categorical variables. Means or medians were compared with a Student's T-test, or with a nonparametric test, as appropriate. Hazard ratios for all-cause mortality were calculated with a Cox regression model. Survival was calculated with a Kaplan-Meier model, and groups were compared with logostic regression. The threshold for statistical significance was set at $p<0.05$. 


\section{Results}

\section{Baseline characteristics}

In our centre, 330 patients underwent a total of 480 interventions with curative intent for HCC. Patients who underwent primary HR, combined HR with intraoperative TA, or OLTx as their primary interventions $(n=154)$ were excluded. The remaining 176 patients underwent primary TA: RFA in 72 patients $(40.9 \%)$, and MWA in 104 patients $(59.1 \%)$ Date of primary TA was January 2015 or later in $52.2 \%$ of cases, and January 2017 or later in $34.7 \%$ of cases. Table 1 shows baseline characteristics. Over $90 \%$ of patients had cirrhosis. Steatohepatitis was the most frequent cause of liver disease, followed by viral hepatitis. At presentation, patients had

Table 1 Patient and tumour characteristics at time of primary thermal ablation (TA)

\begin{tabular}{ll}
\hline Demographics & Patients $(N=176)$ \\
\hline Median age (IQR; range) & 64.5 years; $(58.0-70.8 ;$ \\
Male sex, N (\%) & $12-90)$ \\
Cirrhosis & \\
Yes & $159(90.7 \%)$ \\
Child-Pugh class & \\
A & $151(89.3 \%)$ \\
B & $18(10.7 \%)$ \\
Primary Underlying Liver Disease & \\
(N)ASH & $94(53.4 \%)$ \\
Viral & $53(30.1 \%)$ \\
Metabolic & $6(3.4 \%)$ \\
Cholestatic & $5(2.8 \%)$ \\
Cryptogenic cirrhosis & $8(4.5 \%)$ \\
Other/unknown & $10(5.7 \%)$ \\
Tumour characteristics & \\
Median diameter of largest tumour (IQR; & $2.7 \mathrm{~cm}(2.0-3.3 ; 0.5-8.0)$ \\
range) & \\
Number of tumours & \\
1 & $126(71.6 \%)$ \\
2 & $42(23.9 \%)$ \\
3 & $6(3.4 \%)$ \\
BCLC stage & $2(1.1 \%)$ \\
Very early & \\
Early & \\
Intermediate & \\
Type of TA & \\
RFA & \\
MWA & \\
AFP level & \\
\hline
\end{tabular}

one to four detectable tumours on pre-operative imaging. At presentation, 50 patients had multiple tumours $(28.4 \%)$. Median tumour size was $2.7 \mathrm{~cm}$; a quarter of patients had at least one tumour $\geq 3.3 \mathrm{~cm}$ in diameter.

\section{Recurrence and repeat interventions after primary TA}

After primary TA, routine CT on day 7 showed satisfactory ablation margins in 161 patients $(91.5 \%)$. The remaining 15 patients $(8.5 \%)$ all underwent successful completion TA, which were not counted as repeat ablations [12]. Median follow-up was 30.9 months (IQR 13.4-66.0). During followup, recurrences were detected in 99 of 176 patients $(56.3 \%)$, of which 85 were intrahepatic $(85.9 \%)$. In patients who were treated with repeat TA for recurrence after primary TA, the median time to recurrence was 13 months (IQR 6-23 months). Patient-specific treatment paths and outcomes are shown in Fig. 1 (for more details, fully quantitative data can be found in the Supplementary Material). Of 85 patients with intrahepatic recurrence, 76 patients $(89.4 \%)$ underwent between 1 to 4 repeat interventions (TA, HR, OLTx) with curative intent. Not counting 19 patients $(10.8 \%)$ who never had the chance to develop recurrent $\mathrm{HCC}$ due to death from other causes within 6 months of their last intervention, 116 of 176 patients $(65.9 \%)$ remained recurrence-free during a median follow-up of 27.5 months after their last intervention. At any point in time after primary TA, a total of $41(23.3 \%)$ patients developed recurrences that could not be treated with repeat TA, HR or OLTx. In this group, 21 patients $(51.2 \%)$ underwent transarterial chemoembolisation (TACE) or selective intra-arterial radiotherapy (SIRT) for locally advanced disease, 13 patients $(31.7 \%)$ were treated with sorafenib for metastatic disease, and the remaining 7 patients $(17.1 \%)$ received best supportive care. A total of 37 patients $(21.0 \%)$ underwent OLTx at any point in time after primary TA; and three patients (1.7\%) underwent HR. In two patients (1.1\%), both after OLTx following primary TA, resection of a late, solitary distant metastasis was performed, after which they remained recurrence-free. Excluding 37 patients who underwent OLTx after one or more sessions of TA, 139 "TA-only" patients remained. Not counting 16 patients (11.5\%) with non-HCC-related death within 6 months after their last intervention, 86 of 139 TAonly patients $(61.9 \%)$ remained recurrence-free following their last intervention. Eventually, 37 of 139 patients $(26.6 \%)$ developed incurable recurrence.

\section{Survival}

At the end of follow-up, 65 patients (36.9\%) had died. Causes of death were HCC in 27 cases, (41.5\% of deaths), 
Repeat treatments and outcomes following primary thermal ablation (TA) for hepatocellular carcinoma

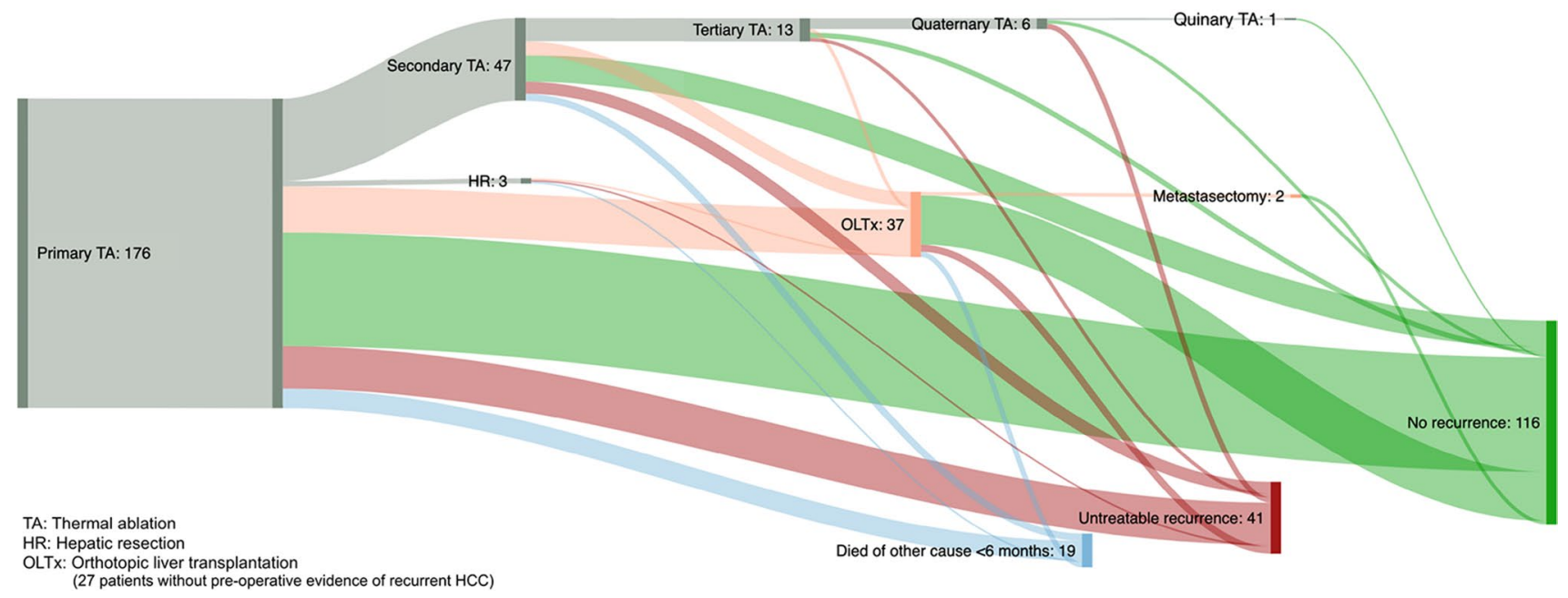

Fig. 1 Patient-specific treatment paths following primary thermal ablation (TA) for HCC. The order of, and outcomes after subsequent re-interventions are shown. More highly detailed, fully quantitative data can be found in the flow chart in Supplementary Materials

progression of liver disease without signs of recurrent HCC in 11 cases $(16.9 \%)$, and other causes in 27 cases $(41.5 \%)$. Median OS after primary TA was 96.0 months (95\% CI 41.0-150.9). At 5 years, OS was 54.0\%. Median RFS after TA was 19.0 months. Median DSS was not reached. At 5 years, DSS was $73.8 \%$. Table 2 shows hazard ratios for death for different patient and tumour related factors. Age, number of tumours and AFP levels were associated with mortality.

\section{Primary TA versus repeat TA}

After primary TA, 49 of 176 patients (27.8\%) underwent up to four sessions of repeat TA, resulting in a total of 243 ablation sessions. During 67 sessions of repeat TA, patients

Table 2 Correlations of different clinical features with all-cause mortality

\begin{tabular}{llllll}
\hline & \multicolumn{2}{l}{ Univariate } & & \multicolumn{2}{l}{ Multivariate } \\
\cline { 2 - 3 } \cline { 6 - 7 } & HR & $p$ & & HR & $p$ \\
\hline Age & 1.029 & $\mathbf{0 . 0 3 6}$ & & 1.029 & $\mathbf{0 . 0 6 3}$ \\
Cirrhosis & 2.113 & 0.207 & & N/A & N/A \\
HBV & 0.743 & 0.369 & & N/A & N/A \\
HCV & 0.840 & 0.566 & & N/A & N/A \\
Alcohol abuse & 1.075 & 0.258 & & N/A & N/A \\
MELD score & 1.077 & 0.121 & & N/A & N/A \\
$N$ of tumours at baseline & 1.492 & $\mathbf{0 . 0 2 0}$ & & 1.497 & $\mathbf{0 . 0 4 1}$ \\
Baseline tumour diameter & 1.020 & 0.166 & & N/A & N/A \\
aFP level at baseline & 1.001 & $<\mathbf{0 . 0 0 1}$ & & 1.001 & $<\mathbf{0 . 0 0 1}$ \\
MWA (vs. RFA) & 1.050 & 0.848 & & N/A & N/A \\
\hline
\end{tabular}

Bold emphasis on $p$ values reaching or approaching statistical significance were treated for solitary tumours in 52 sessions $(77.6 \%$, versus $71.6 \%$ at primary TA; $p=0.218)$. Mean tumour diameter during repeat TA $(2.8 \mathrm{~cm}$; SD 1.3, range $0.8-3.2)$ was comparable to mean tumour diameter at primary TA $(2.9 \mathrm{~cm}$; SD 1.2; range $0.5-8.0 ; p=0.810$ ). Figure 2 shows $\mathrm{OS}$ and RFS after primary TA, after one session of repeat TA, and after multiple sessions of repeat TA. We found no statistically significant differences in OS calculated from the date of the last-performed TA session, neither when comparing between all three groups separately (primary TA only; one session of repeat TA; two or more sessions of repeat TA: $p=0.433$, Fig. $2 \mathrm{a}$ ), nor when combining all patients with repeat TA into one group ( $p=0.288$, Fig. $2 b$ ). RFS, however, was shorter after repeat TA, compared to patients who underwent only primary TA ( $p=0.005$, Fig. $2 c)$. Quantitative survival times, calculated from primary and repeat interventions, are indicated in Fig. 2d.

Incidence and severity of complications (graded according to Clavien-Dindo [10]) were comparable following primary and repeat TA sessions $(p=0.840)$. After primary TA, there were no complications in 132 patients $(75.0 \%)$, grade 1-2 complications in 33 patients (18.8\%), and complications graded 3 and above in 11 patients (6.3\%). After 67 sessions of repeat TA, there were no complications following 56 sessions (83.6\%), grade 1-2 complications following 10 sessions (14.9\%), and a grade 3 complication following 1 session (1.5\%). TA-related 30-day mortality was $1.2 \%$.

\section{Primary TA followed by OLTx versus no subsequent OLTX}

After primary TA $(n=176), 55$ patients were screened and accepted for the OLTx waitlist. After having been accepted, 


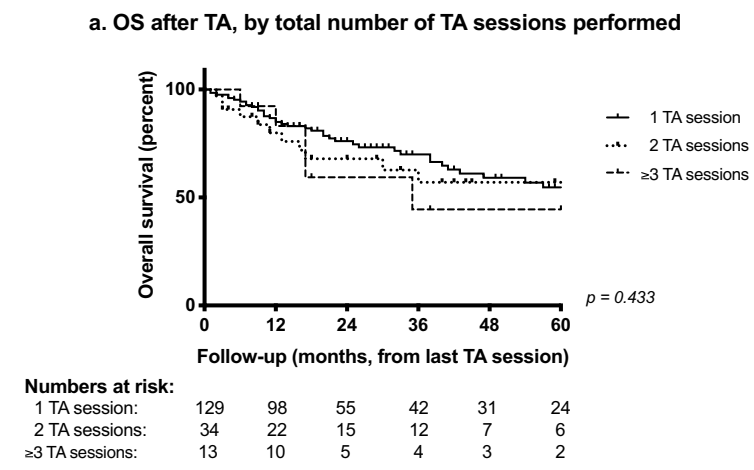

c. RFS after last TA, by total number of TA sessions performed

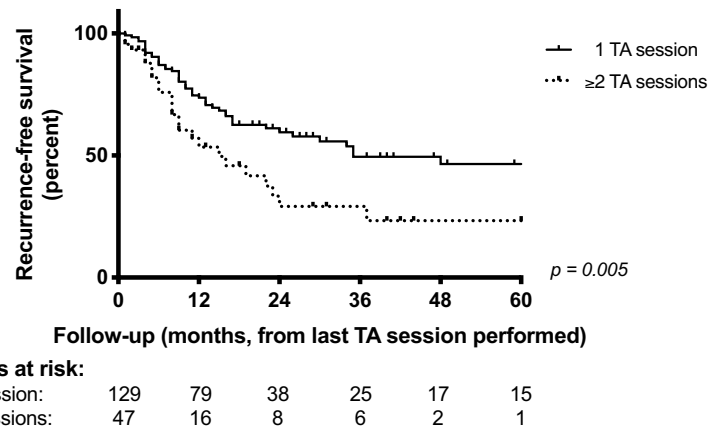

Fig. 2 a Overall survival, calculated from the last session of thermal ablation (TA) performed, stratified by total number of TA sessions performed (1, 2 or $\geq 3$ ). b Overall survival, calculated from the last session of TA performed, comparing those patients who underwent only primary TA to patients who underwent $\geq 2$ sessions of TA. c Recurrence-free survival, calculated from the last session of TA per-

six patients refrained from active waitlisting due to various non-medical reasons, leaving 49 patients who were awaiting a donor liver at any point in time. Reasons not to screen patients for OLTx were age $>70$ years in 53 cases (of 121 non-waitlisted patients, $43.8 \%$ ), unfavourable oncology in $10(8.3 \%)$, prohibitive comorbidity in $9(7.4 \%)$, patient or physician preference in $25(20.7 \%)$, ongoing alcohol abuse in $8(6.6 \%)$, and other reasons in $16(13.2 \%)$. Eventually, 37 of 49 actively waitlisted patients $(75.5 \%)$ underwent OLTx. There was no pre-operative evidence of recurrent or residual HCC before OLTx in 27 patients (73.0\%). A comparison of clinicopathological characteristics of transplanted versus non-transplanted patients is shown in Table 3. Transplanted patients were younger, more often male, $\mathrm{HCV}$ positive and universally cirrhotic. Survival for both groups is shown in Fig. 3. Median and 5-year OS were better for patients who b. OS after TA, by total number of TA sessions performed (single vs. multiple)

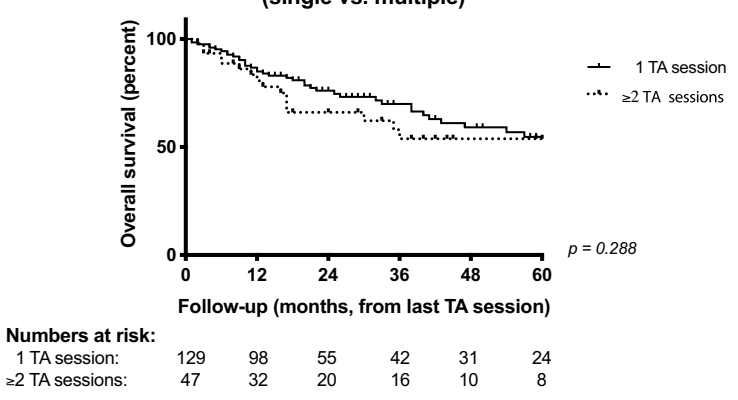

d. OS and RFS, counted from first, second and last TA session
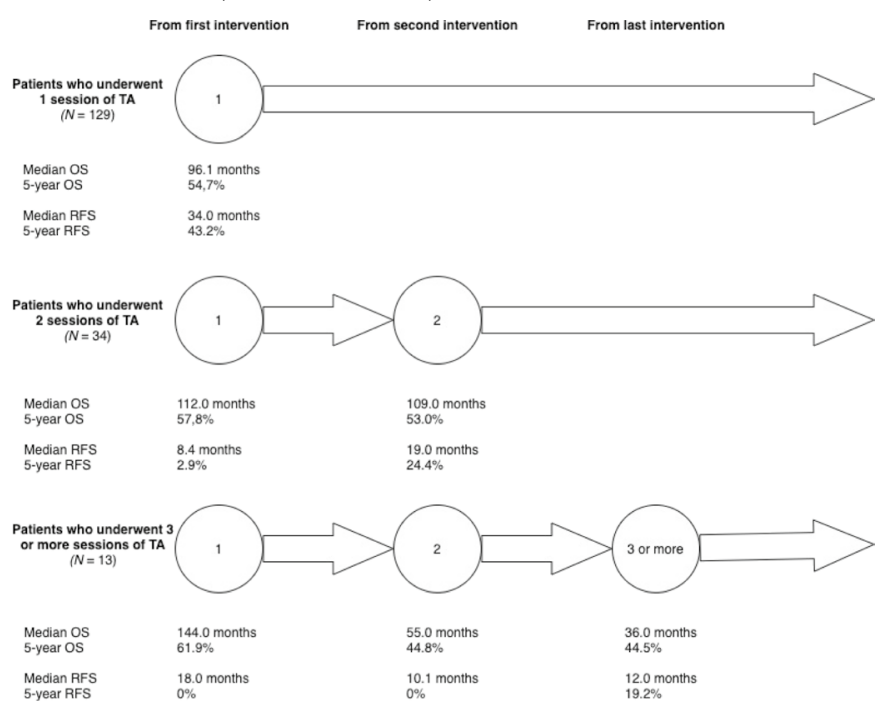

formed, comparing those patients who underwent only primary TA to patients who underwent $\geq 2$ sessions of TA. d Overall and recurrencefree survival, calculated from the date of primary TA (1), from the first session of repeat TA (2), and from the last session of repeat TA ( 3 or more). Values are shown separately for patients who underwent one, two, or more sessions of TA

underwent OLTx (137 vs. 54 months; $74.7 \%$ vs. $45.0 \%$; $p=0.011$, Fig. 3a). To correct for both lead-time bias due to the interval between TA and OLTx, and selection bias due to higher age, more severe comorbidity, and more unfavourable oncology for the non-OLTx group, we calculated survival for an "intention-to-perform-OLTX" group, consisting of all patients who had at any point in time been actively waitlisted with the intention to perform OLTx after primary TA $(n=49)$, and compared them to an "eligible, non-waitlisted" group $(n=55)$. This latter group consisted of those patients who would have qualified for OLTx on medical grounds (see methods), but were never actively waitlisted due to other reasons. Groups had comparable median age $(p=0.835)$, Child-Pugh Class $(p=0.305), \operatorname{AFP}(p=0.840)$, tumour size $(p=0.426)$, and number of tumours $(p=0.724)$ at primary TA. OS was comparable between these groups 
Table 3 Clinicopathological characteristics of patients who underwent orthotopic liver transplantation (OLTx) after primary thermal ablation (TA), versus those who did not

\begin{tabular}{llll}
\hline Parameters at time of primary TA & $\begin{array}{l}\text { Never OLTx } \\
(n=139)\end{array}$ & $\begin{array}{l}\text { Eventual OLTx } \\
(n=37)\end{array}$ & \\
\hline Median age (IQR) & $66.5(59.0-71.3)$ & $59.5(53.7-65.0)$ & $\boldsymbol{p}=\mathbf{0 . 0 0 2}$ \\
Male sex & $107(77.0 \%)$ & $35(94.6 \%)$ & $\boldsymbol{p}=\mathbf{0 . 0 3 1}$ \\
Median diameter of primary tumour (IQR) & $2.7 \mathrm{~cm}(2.1-3.3)$ & $2.8 \mathrm{~cm}(2.0-3.6)$ & $p=0.344$ \\
Number of tumours at primary intervention & & & $p=0.304$ \\
1 & $97(69.8 \%)$ & $29(78.4 \%)$ & \\
2 or more & $42(30.2 \%)$ & $8(21.6 \%)$ & \\
Cirrhosis & $122(87.8 \%)$ & $37(100 \%)$ & $\boldsymbol{p}=\mathbf{0 . 0 1 8}$ \\
Child-Pugh B & $13(9.7 \% ; 5$ missing) & $5(14.3 \% ; 2$ miss- & $p=0.652$ \\
HCV & $19(13.7 \%)$ & $13(35.1 \%)$ & $\boldsymbol{p}=\mathbf{0 . 0 0 6}$ \\
Median AFP at baseline & 7.2 & 7.9 & $p=0.864$ \\
\hline
\end{tabular}

Bold emphasis on $p$ values reaching or approaching statistical significance

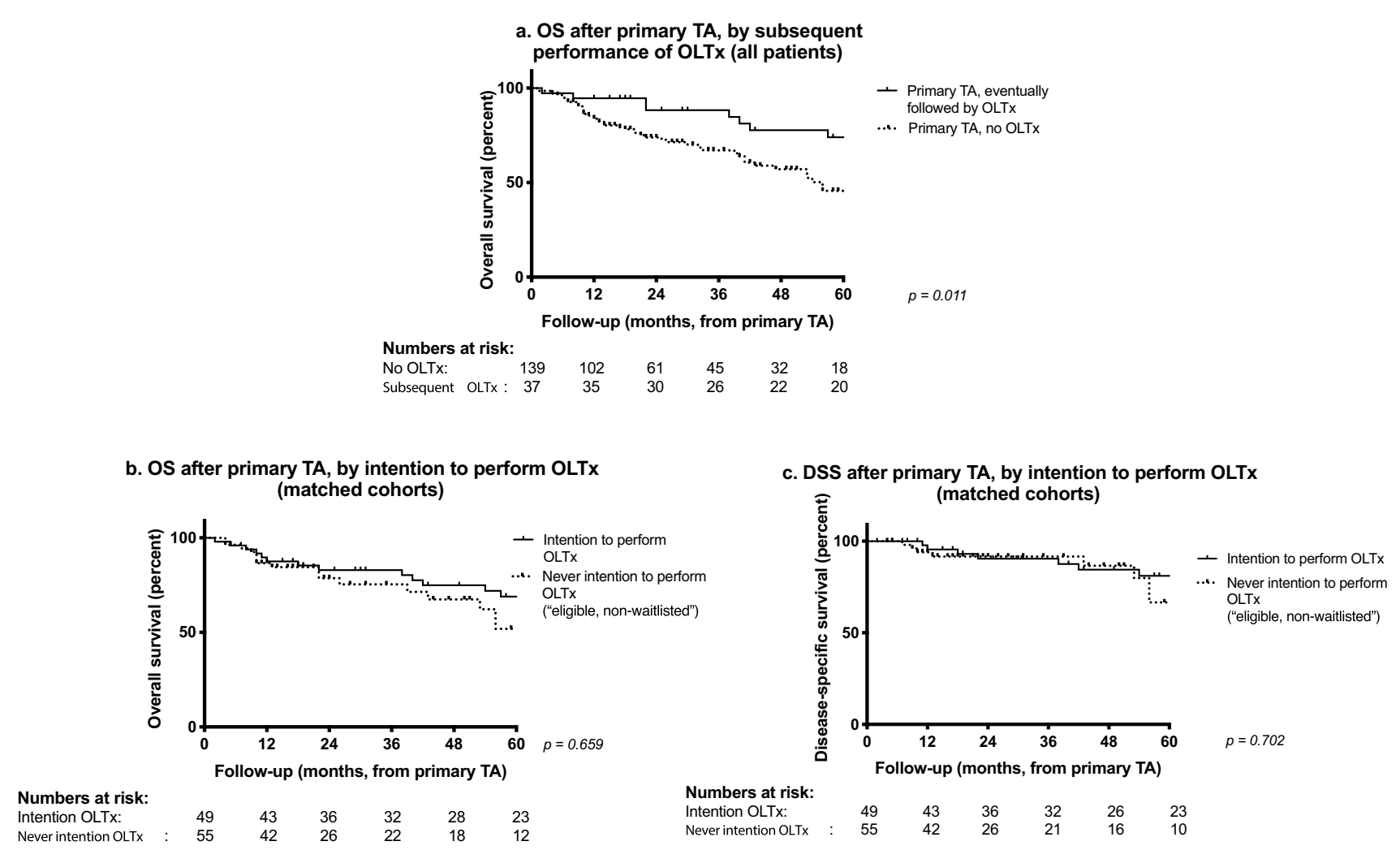

Fig. 3 a Overall survival calculated from the date of primary thermal ablation (TA), comparing all patients who subsequently underwent orthotopic liver transplantation (OLTx) to those who did not. b Overall survival calculated from the date of primary TA, comparing those patients who were actively waitlisted for OLTx ("intention-

(median 121.6 months vs. 109.8 months; 5-year OS 68.7\% vs. $52.9 \%$; $p=0.659$, Fig. 3 b), as was DSS $(81.1 \%$ vs. $66.7 \%$ at 5 years, $p=0.702$, Fig. $3 c$ ). to-perform OLTx") to a matched cohort of patients who would have qualified OLTx, but were not waitlisted due to either patient or physician preference, or ongoing alcohol abuse ("eligible, non-waitlisted"). c Disease-specific survival calculated from the date of primary TA, comparing the same matched groups as in $\mathrm{B}$

\section{Discussion}

We found TA to be a reliable, repeatable treatment modality for HCC. After primary TA, almost half of patients 
never developed clinically apparent recurrence during follow-up. Most recurrences could be treated with curative intent, either with repeat TA or with OLTx. Some patients underwent numerous sessions of TA without developing an incurable recurrence. Even though RFS was shorter after repeat TA than after primary TA, we found no difference in OS. In fact, after matching patients for baseline oncological characteristics, comorbidity, and age, we found that OS after TA without ever being waitlisted for OLTx was comparable to OS after TA as a step-up to OLTx. Our findings imply the existence of a group of patients who do remarkably well with TA alone, some despite developing multiple recurrences. There is almost no data on outcomes after repeated sessions of TA. A literature search on PubMed-listed studies from January 2015 to 2021 (search terms: ((HCC[Title]) OR (hepatocellular carcinoma[Title])) AND ((ablation[Title]) OR (RFA[Title])) AND ((redo[Title]) OR (recurrence[Title]) OR (repeat[Title]) OR (recurrent[Title])), yielded 104 hits. Manual screening revealed two studies which reported on survival after repeat TA following primary TA $[13,14]$. Both were performed in Asian populations, with comparatively young patients (median age 50 [13], and 60 [14]), with almost exclusively viral hepatitis-induced HCC. One study reported a number of non-cirrhotic patients above $30 \%$ [13]. Therefore, we believe these studies are not generalisable outside of their highly specific demographic contexts.

We found a median OS after primary TA of 96.0 months, and a 5 -year OS of $54.1 \%$. A widely varying 5 -year OS after TA has been reported in the literature, mostly around 40-60\% [15-17], (outliers in selected populations 30\%-80\% [18-20]). It must be noted that our patient population is relatively old (median 64.5 years; $25 \%$ of patients $\geq 70$ years), while most studies had a median patient age around 55 years $[17,20]$, with only sporadic studies in a comparable age group [16, 19]. Furthermore, tumours in our study were relatively large (median $2.7 \mathrm{~cm}, 25 \%$ of tumours $\geq 3.3 \mathrm{~cm}$ ), whereas many studies excluded tumours $>3 \mathrm{~cm}[15,21]$, or had such tumours only in small numbers [20]. Lastly, some authors excluded patients with multiple tumours [21] or neoadjuvant therapy [19].

As causes of death are variable in patients with chronic liver disease, DSS may be a better measure of oncologic results, especially when comparing results across different populations. Unfortunately, few studies report DSS. Our 5 -year DSS of $73.8 \%$ after TA is high compared to the literature [22, 23].

Our finding that $13.6 \%$ of patients developed incurable recurrence following primary TA ( $24 \%$ of first recurrences) appears to be in contrast to the suggestion by Doyle et al. that $42 \%$ of recurrences are beyond Milan criteria, when analysing a series of smaller, unifocal primary
HCC's [21]. Despite some patients undergoing up to four sessions of repeat TA, only $23.3 \%$ of our patients eventually developed a recurrence unamenable to repeat TA or OLTx. These results might be because of the relatively high technical success rate of our TA workflow, and due to our strict follow-up protocol, which allows us to detect recurrences early on.

Over the past two decades, TA has established itself as an effective treatment option for HCC. Little evidence supports its formerly assumed inferiority to HR [19, 24]. Indeed, our DSS after TA is not inferior to rates reported after HR [25, 26]. More uncertain is the optimal relationship between TA and OLTx. On the one hand, OLTx leads to the best recurrence-free survival [5], with some authors proclaiming OLTx to be the only curative treatment [27], and others suggesting that locoregional treatments for HCC should be thought of as "a bridge to nowhere" [28]. By removing the preneoplastic cirrhotic liver parenchyma in its entirety, OLTx adresses the driving factor behind HCC recurrence [25]. On the other hand, OLTx is a major procedure with a lasting impact on patients' lives [6], and is restricted by a shortage of donor organs. It is therefore imperative that we put these scarce resources to optimal use.

Our findings support a more nuanced view of the role of TA in early and very early HCC than hitherto proposed by many. Until now, clinicians have focused on identifying patients who have the lowest risk of posttransplant recurrence [29], but this is only one side of the picture. Ideally, we should reserve OLTx for patients who would have bad outcomes with locoregional therapy alone [30].

A shortcoming of our study is its observational nature. This brings with it an inherent danger of confounding variables and selection bias, for which it is impossible to fully correct. Furthermore, in some subgroup analyses, smaller numbers may have obscured underlying correlations. The heterogeneity of this patient population makes a sound prospective analysis beyond the first treatment modality extremely difficult.

In conclusion, we found that $\mathrm{TA}$ is an effective treatment modality both for primary and recurrent HCC. Although many patients develop recurrences during follow-up, we demonstrated that most can be treated with repeat TA. There is a group of patients with HCC who survive many years with TA, without ever undergoing OLTx. In matched cohorts, TA without waitlisting for OLTx was not inferior to TA with the intention to perform OLTx. Therefore, it is imperative that we find a way to differentiate between those patients who would do well with TA alone, and those who will require OLTx due to a high risk of untreatable tumour recurrence. This way, we can put scarce donor organs to best use. It is beyond any doubt that if a "TA-first" approach is taken, intensive follow-up is crucial. 
Supplementary Information The online version contains supplementary material available at https://doi.org/10.1007/s00330-021-08515-3.

Added value of this study - It is the first study reporting on the impact of repeat TA for recurrent HCC after primary TA in a non-Asian population of cirrhotic patients.

- It is the first study comparing results after primary and repeat

TA, Showing that survival outcomes are comparable.

- It is the first study reporting outcomes after several sessions of repeat TA. We reconstructed patient-specific treatment paths, demonstrating that even though many patients indeed develop recurrence after primary TA, these can most often be managed with repeat TA. Some patients do well despite multiple recurrences.

- It is, to our knowledge, the first study that compares outcomes after TA with the intention to perform OLTx to a TA-only approach, correcting for inherent bias and confounders (age, comorbidity and tumour stage). In our intention-to-treat analysis, we compared two matched cohorts of patients. The first cohort consisted of patients eligible and waitlisted for OLTx, and the second consisted of patients who would have been eligible for OLTx, but were never waitlisted. This analysis revealed the existence of a group of people who will do well without ever undergoing OLTx.

Implications of all the available evidence - Repeat TA can be an effective therapy and should be considered for recurrent HCC following primary TA.

- Our findings suggest that in patients with preserved liver function, TA should be considered as an alternative to early OLTx. A "TA-only" approach may be reasonable in certain groups of patients with HCC.

- Future research should focus on finding predicting factors that identify those patients who are likely to do well with TA alone, and those who will need OLTx because of a high risk of disease progression despite adequate initial locoregional control.

Funding The authors state that this work has not received any funding.

\section{Declarations}

Guarantor The scientific guarantor of this publication is K.P. de Jong, Hepato-Pancreato-Biliary surgeon, transplant surgeon, and head of the locoregional treatment team of the Department of Hepato-PancreatoBiliary Surgery and Liver Transplantation at the University of Groningen.

Conflict of Interest The authors of this manuscript declare no relationships with any companies whose products or services may be related to the subject matter of the article.

Statistics and Biometry No complex statistical methods were necessary for this paper.

Informed Consent Written informed consent was not required for this study because of its retrospective nature.

Ethical Approval Institutional Review Board approval was not required because of the retrospective nature of our study.
Methodology

- retrospective

- observational

- performed at one institution

Open Access This article is licensed under a Creative Commons Attribution 4.0 International License, which permits use, sharing, adaptation, distribution and reproduction in any medium or format, as long as you give appropriate credit to the original author(s) and the source, provide a link to the Creative Commons licence, and indicate if changes were made. The images or other third party material in this article are included in the article's Creative Commons licence, unless indicated otherwise in a credit line to the material. If material is not included in the article's Creative Commons licence and your intended use is not permitted by statutory regulation or exceeds the permitted use, you will need to obtain permission directly from the copyright holder. To view a copy of this licence, visit http://creativecommons.org/licenses/by/4.0/.

\section{References}

1. Ferlay J, Soerjomataram I, Dikshit R et al (2015) Cancer incidence and mortality worldwide: Sources, methods and major patterns in GLOBOCAN 2012. Int J Cancer 136:E359-E386. https://doi.org/ 10.1002/ijc. 29210

2. Wallace MC, Preen D, Jeffrey GP, Adams LA (2015) The evolving epidemiology of hepatocellular carcinoma: A global perspective. Expert Rev Gastroenterol Hepatol 9:765-779. https://doi.org/10. 1586/17474124.2015.1028363

3. Crocetti L, Bargellini I, Cioni R (2017) Loco-regional treatment of HCC: Current status. Clin Radiol 72:626-635. S00099260(17)30041-7 [pii]

4. Rich NE, Yopp AC, Singal AG (2017) Medical management of hepatocellular carcinoma. J Oncol Pract 13:356-364. https://doi. org/10.1200/JOP.2017.022996

5. Kulik L, Heimbach JK, Zaiem F et al (2018) Therapies for patients with hepatocellular carcinoma awaiting liver transplantation: A systematic review and meta-analysis. Hepatology 67:381-400. https://doi.org/10.1002/hep.29485

6. Damaskos C, Kaskantamis A, Garmpis N et al (2019) Intensive care unit outcomes following orthotopic liver transplantation: Single-center experience and review of the literature. G Chir 40:463-480. 9126 [pii]

7. Pommergaard HC, Rostved AA, Adam R et al (2018) Locoregional treatments before liver transplantation for hepatocellular carcinoma: A study from the european liver transplant registry. Transpl Int. https://doi.org/10.1111/tri.13123

8. Nault JC, Sutter O, Nahon P, Ganne-Carrie N, Seror O (2018) Percutaneous treatment of hepatocellular carcinoma: State of the art and innovations. J Hepatol 68:783-797

9. Bale R, Schullian P, Eberle G et al (2019) Stereotactic radiofrequency ablation of hepatocellular carcinoma: A histopathological study in explanted livers. Hepatology 70:840-850. https://doi.org/ 10.1002/hep.30406

10. Dindo D, Demartines N, Clavien PA (2004) Classification of surgical complications: A new proposal with evaluation in a cohort of 6336 patients and results of a survey. Ann Surg 240:205-213. 00000658-200408000-00003 [pii]

11. de Jong KP, Ruiter SJS, Pennings JP (2019) Stereotactic image guided microwave ablation of HCC: A step forward and still a long way to go. Liver Int 39(10):1798-1800

12. Ahmed M, Solbiati L, Brace CL et al (2014) Image-guided tumor ablation: Standardization of terminology and reporting criteria--a 
10-year update. J Vasc Interv Radiol 25:1691-705.e4. S10510443(14)00845-8 [pii]

13. Wang X, Liang H, Lu Z (2020) Efficacy of transarterial chemoembolization compared with radiofrequency ablation for the treatment of recurrent hepatocellular carcinoma after radiofrequency ablation. Minim Invasive Ther Allied Technol 29:344-352. https://doi.org/10.1080/13645706.2019.1649286

14. Wang Y, Liao Y, Liu W et al (2021) Surgical resection versus re-ablation for intrahepatic recurrent hepatocellular carcinoma after initial ablation therapy. Dig Surg 38:46-57. https://doi.org/ $10.1159 / 000511157$

15. Facciorusso A, Serviddio G, Muscatiello N (2016) Local ablative treatments for hepatocellular carcinoma: An updated review. World J Gastrointest Pharmacol Ther 7:477-489. https://doi.org/ 10.4292/wjgpt.v7.i4.477

16. Zhao WJ, Zhu GQ, Wu YM, Wang WW, Bai BL (2019) Comparative effectiveness of radiofrequency ablation, surgical resection and transplantation for early hepatocellular carcinoma by cancer risk groups: Results of propensity score-weighted analysis. Onco Targets Ther 12:10389-10400. https://doi.org/10.2147/OTT. S224809

17. Huang J, Yan L, Cheng $\mathrm{Z}$ et al (2010) A randomized trial comparing radiofrequency ablation and surgical resection for HCC conforming to the milan criteria. Ann Surg 252:903-912. https:// doi.org/10.1097/SLA.0b013e3181efc656

18. Iida H, Aihara T, Ikuta S, Yamanaka N (2014) Comparative study of percutaneous radiofrequency ablation and hepatic resection for small, poorly differentiated hepatocellular carcinomas. Hepatol Res 44:E156-E162. https://doi.org/10.1111/hepr.12264

19. Mohkam K, Dumont PN, Manichon AF et al (2018) No-touch multibipolar radiofrequency ablation vs. surgical resection for solitary hepatocellular carcinoma ranging from 2 to $5 \mathrm{~cm}$. J Hepatol. S0168-8278(18)30057-6 [pii]

20. Lee HW, Lee JM, Yoon JH et al (2018) A prospective randomized study comparing radiofrequency ablation and hepatic resection for hepatocellular carcinoma. Ann Surg Treat Res 94:74-82. https:// doi.org/10.4174/astr.2018.94.2.74

21. Doyle A, Gorgen A, Muaddi H et al (2019) Outcomes of radiofrequency ablation as first-line therapy for hepatocellular carcinoma less than $3 \mathrm{~cm}$ in potentially transplantable patients. J Hepatol 70:866-873. S0168-8278(19)30001-7 [pii]
22. Jiang YQ, Wang ZX, Deng YN, Yang Y, Wang GY, Chen GH (2019) Efficacy of hepatic resection vs. radiofrequency ablation for patients with very-early-stage or early-stage hepatocellular carcinoma: A population-based study with stratification by age and tumor size. Front Oncol 9:113. https://doi.org/10.3389/fonc. 2019.00113

23. Yue YY, Zhou WL (2020) Hepatic resection is associated with improved long-term survival compared to radio-frequency ablation in patients with multifocal hepatocellular carcinoma. Front Oncol 10:110. https://doi.org/10.3389/fonc.2020.00110

24. Gui CH, Baey S, D'cruz RT, Shelat VG (2020) Trans-arterial chemoembolization + radiofre-quency ablation versus surgical resection in hepatocellular carcinoma - A meta-analysis. Eur J Surg Oncol 46:763-771. S0748-7983(20)30004-4 [pii]

25. Sasaki K, Shindoh J, Margonis GA et al (2017) Effect of background liver cirrhosis on outcomes of hepatectomy for hepatocellular carcinoma. JAMA Surg 152:e165059. https://doi.org/10. 1001/jamasurg.2016.5059

26. Yang A, Xiao W, Chen D et al (2018) The power of tumor sizes in predicting the survival of solitary hepatocellular carcinoma patients. Cancer Med 7:6040-6050. https://doi.org/10.1002/cam4. 1873

27. Kollmann D, Selzner N, Selzner M (2017) Bridging to liver transplantation in HCC patients. Langenbecks Arch Surg 402:863-871. https://doi.org/10.1007/s00423-017-1609-2

28. Tan CHN, Yu Y, Tan YRN et al (2018) Bridging therapies to liver transplantation for hepatocellular carcinoma: A bridge to nowhere? Ann Hepatobiliary Pancreat Surg 22:27-35. https://doi. org/10.14701/ahbps.2018.22.1.27

29. Cucchetti A, Serenari M, Sposito C et al (2020) Including mRECIST in the metroticket 2.0 criteria improves prediction of hepatocellular carcinoma-related death after liver transplant. J Hepatol 73:342-348. S0168-8278(20)30177-X [pii]

30. Mehta N, Yao FY (2019) What are the optimal liver transplantation criteria for hepatocellular carcinoma? Clin Liver Dis (Hoboken) 13:20-25. https://doi.org/10.1002/cld.793

Publisher's note Springer Nature remains neutral with regard to jurisdictional claims in published maps and institutional affiliations. 